\title{
Two structure theorems for homeomorphism groups
}

\author{
A. R. Vobach
}

Let $H(C)$ be the group of homeomorphisms of the Cantor set, $C$, onto itself. Let $p: C \rightarrow M$ be a (continuous) map of $C$ onto a compact metric space $M$, and let $G(p, M)$ be $\{h \in H(C) \mid \forall x \in C, p(x)=p h(x)\} . G(p, M)$ is a group. The map $p: C \rightarrow M$ is standard, if for each $(x, y) \in C \times C$ such that $p(x)=p(y)$, there is a sequence $\left\{x_{n}\right\}_{n=1}^{\infty} \subset C$ and a sequence $\left\{h_{n}\right\}_{n=1}^{\infty} \subset G(p, M)$ such that $x_{n}+x$ and $h_{n}\left(x_{n}\right) \rightarrow y$. Standard maps and their associated groups characterize compact metric spaces in the sense that: Two such spaces, $M$ and $N$, are homeomorphic if and only if, given $p$ standard from $C$ onto $M$, there is a standard $q$ from $C$ onto $N$ for which $G(p, M)=h^{-1} G(q, N) h$, for some $h \in H(C)$. That is, two compact metric spaces are homeomorphic if and only if they determine, via standard maps, the same classes of conjugate subgroups of $H(C)$.

The present note exhibits two natural structure theorems relating algebraic and topological properties: First, if $M=H \cup K \quad(H, K \neq \varnothing)$, compact metric, and $p: C \rightarrow M$ are given, then $G(p, M)$ is isomorphic to a subdirect product of $G(p, M) / S(p, H \backslash K)$ and $G(p, M) / S(p, K \backslash H)$ where, generally, $S(p, N)$ is the normal subgroup of homeomorphisms supported on $p^{-1}(I N)$. Second, given $M$ and $N$ compact metric and $P: M \rightarrow N$ continuous and onto, let $M \neq M-C D_{\alpha}^{*} \neq \varnothing$, where $\left\{D_{\alpha}\right\}_{\alpha \in A}$ is

Received 26 August 1970. 
the collection of non-degenerate preimages of points in $N$. Then there is a standard $p: C \rightarrow M$ such that $f p: C \rightarrow N$ is standard and there is a homomorphism

$$
H: G(p, M) \rightarrow G(f p, N) / S\left(f p, \mathrm{Cl} D_{\alpha}^{*}\right)
$$

\section{Introduction}

The results described in the Abstract appeared in [2]. Later, in [3], it was shown that if $M_{1}$ and $M_{2}$ are compact metric spaces, then there are standard maps $p: C \rightarrow M_{1} \times M_{2}$ and $p_{i}: C \rightarrow M_{i}, i=1,2$, such that $G\left(p, M_{1} \times M_{2}\right)=G\left(p_{1}, M_{1}\right) \cap G\left(p_{2}, M_{2}\right)$.

In some vague sense "dual" to this is the observation: if $M=H \cup K$ where $H \cap K=\varnothing$ and each of $H$ and $K$ is closed, then, for $p: C \rightarrow M$, $G(p, M)$ is the (interior) direct product of the two normal subgroups supported on (the identity on the complement of) the preimages of $H$ and of $K$, respectively.

The first of the following theorems concerns the less fortuitous circumstance in which $H$ and $K$ intersect.

The second theorem, below, grows out of the observation in [2], that if $f: M \rightarrow N$ is continuous and onto, for $M$ and $N$ compact metric, then there is a standard map $p: C \rightarrow M$ such that $f p: C \rightarrow N$ is also standard and $G(p, M) \subset G(f p, N)$. Theorem 2 is an indication, for many sases, of "how much bigger" $G(f p, N)$ need be.

First a simple preparatory lemma:

LEMMA. Given compact metric $M, N \subset M$ and a map $p: C \rightarrow M$, then $S(p, N):=\left\{h \in G(p, M) \mid \forall x \in C \backslash p^{-1}(N), h(x)=x\right\}$ is a normal subgroup of $G(p, M)$.

Proof. Since $S(p, N)$ is obviously a subgroup, we show only normality: if $x \notin p^{-1}(N), h(x) \notin p^{-1}(N)$ for any $h \in G(p, M)$. Hence for $f \in S(p, N)$ and $h \in G(p, M), h^{-1} f h(x)=x$, for $x \notin p^{-1}(N)$; and $h^{-1} f h \in S(p, N)$. 
Note that, while $H(C)$ is simple (see [1]), the groups $G(p, M)$ have many different normal subgroups.

THEOREM 1. Let compact metric $M=H \cup K$ and let $p: C \rightarrow M$ be given. Then $G(p, M)$ is isomorphic to a subdirect product of $G(p, M) / S(p, H \backslash K)$ and $G(p, M) / S(p, K \backslash H)$.

Proof. Clearly, $S(p, H \backslash K) \cap S(p, K \backslash H)$ is the identity subgroup. Hence, by a well known theorem about groups, $G(p, M)$ is isomorphic to a subdirect product of $G(p, M) / S(p, H \backslash K)$ and $G(p, M) / S(p, K \backslash H)$. The isomorphism may be chosen so that the elements of $[G(p, M) / S(p, H \backslash K)] \times[G(p, M) / S(p, K \backslash H)]$ in the isomorph of $G(p, M)$ are precisely those of the form $(g S(p, H \backslash K), g S(p, K \backslash H))$, $g \in G(p, M)$.

Generally, "subdirectness" indicates the necessary coupling between restrictions of $G(p, M)$ - homeomorphisms to preimages of non-separated sets.

THEOREM 2. Let $M$ and $N$ be compact metric spaces and let $f: M+N$ be continuous and onto. Let $M \neq M-C 1 D_{\alpha}^{*} \neq \emptyset$, where $\left\{D_{\alpha}\right\}_{\alpha \in A}$ is the collection of non-degenerate preimages of points in $N$ and $D_{\alpha}^{*}$ is their union. Then there is a standard $p: C \rightarrow M$ such that $f p: C \rightarrow N$ is standard and there is an onto homomorphism $H: G(p, M) \rightarrow G(f p, N) / S\left(f p, C 1 D_{\alpha}^{*}\right)$. If $\mathrm{Cl}_{\alpha}^{*}$ is nowhere dense in $M$, $p$ and $H$ can be chosen so that $H$ is an isomorphism.

Proof. Let $\left\{T_{i}^{1}\right\}_{i=1}^{n(1)},\left\{T_{i}^{2}\right\}_{i=1}^{n(2)}, \ldots$, be a sequence of finite closed covers of $M$ with the properties:

1) mesh of $\left\{T_{i}^{k}\right\}_{i=1}^{n(k)}$ and of $\left\{f\left(T_{i}^{k}\right)\right\}_{i=1}^{n(k)}<1 / k$;

2) $T_{i}^{k} \cap T_{j}^{k} \neq \emptyset \quad\left(\right.$ and hence $\left.f\left(T_{i}^{k}\right) \cap f\left(T_{j}^{k}\right) \neq \emptyset\right)$ is the union of two or more elements of $\left\{T_{i}^{k+1}\right\}_{i=1}^{n(k+1)}\left(\left\{f\left(T_{i}^{k+1}\right)\right\}_{i=1}^{n(k+1)}\right) ; \quad$ and 
3) for each $k, \mathrm{Cl}\left(M-\mathrm{Cl} D_{\alpha}^{*}\right)$ is the union of elements of $\left\{T_{i}^{k}\right\}_{i=1}^{n(k)}$, each of which is the closure of an open set in $M$.

Let the $T_{i}^{k}$ 's be listed so that the closures of the open sets which cover $\mathrm{Cl}\left(M-\mathrm{Cl} D_{\alpha}^{*}\right)$ occur first: $T_{i}^{k} \subset \mathrm{Cl}\left(M-\mathrm{Cl} D_{\alpha}^{*}\right), \quad 1 \leq i \leq N(k)<n(k)$.

Now divide the interval $[0,1]$ into $2 n(1)$ equal subintervals, labeling every second one of these, end points included, as $E_{1}^{l}, E_{2}^{l}, \ldots, E_{n(1)}^{l}$. Given the interval $E_{i(1), i(2), \ldots, i(k)}^{k}$, where $i(1), \ldots, i(k)$ is such that $T_{i(1)}^{1} \supset T_{i(2)}^{2} \supset \ldots \supset T_{i(1)}^{k}$, divide it into $2 m(i(k))$ equal subintervals where $m(i(k))$ is the number of elements of $\left\{T_{i}^{k+1}\right\}_{i=1}^{n(k+1)}$ contained in $T_{i(k)}^{k}$. Denote every second one of these by

$$
\begin{aligned}
E_{i(1), i(2), \ldots, i(k), j(1)}^{k+1}, E_{i(1), i(2), \ldots, i(k), j(2)}^{k+1}, & \\
& \ldots, E_{i(1), i(2), \ldots, i(k), j(m(i(k))),}^{k+1},
\end{aligned}
$$

where the $j(r)$ 's are the subscripts of the elements of the $(k+1)-$ st cover which are contained in $T_{i(k)}^{k}$.

$$
\text { Set } c=\prod_{k=1}^{\infty}\left(U E_{i(1)}^{k}, \ldots, i(k)\right) \text { for all sequences } i(1), \ldots, i(k)
$$

for which $T_{i(1)}^{1} \supset T_{i(2)}^{2} \supset \ldots \supset T_{i(k)}^{k} \cdot C$ is a Cantor set. Let $F_{n}(x)=T_{i(n)}^{n}$ for $x \in C \cap E_{i(1), \ldots, i(n)}^{n}$, where $i(1), \ldots, i(n)$ is such that $T_{i(1)}^{\perp} \supset \ldots \supset T_{i(n)}^{n}$. Let $p(x)=\bigcap_{n=1}^{\infty} F_{n}(x)$, a (continuous) map of $C$ onto $M$. Observe, as in [2], that $f p: C \rightarrow N$ is also standard by condition 2) above.

(The routine constructions of $C$ and $p$ have been repeated to 
permit an identification of the parts of $C$ with which we deal next.)

Letting

$$
C_{1}=\bigcup_{k=1}^{\infty} \underset{1 \leq i(k) \leq N(k)}{U}\left(C \cap E_{i(1)}^{k}, \ldots, i(k)\right)=\underset{1 \leq i(1) \leq N(1)}{U}\left(C \cap E_{i(1)}^{1}\right) \text {, }
$$

and $C_{2}=C \backslash C_{1}$, observe that $h\left(C_{1}\right)=C_{1}$ by the third condition above, on the covers of $M$ for $h \in G(p, M)$. Otherwise, points in the fp-preimage of $M-\mathrm{Cl} D_{\alpha}^{*}$ would be carried onto points of its complement. Since $C_{1}$ is both open and closed, each homeomorphism in $G(p, M)$ may be expressed, uniquely except for order, as the product of homeomorphisms supported on (the identity on the complement of ) each of. $C_{1}$. and $C_{2}$. Thus, $G(p, M)$ is the interior direct product of its normal subgroups $G_{1}$ and $G_{2}$, the homeomorphisms supported on $C_{1}$ and $C_{2}$, respectively.

Likewise, $G(f p, N)$ is the interior direct product of its normal subgroups of homeomorphisms supported on each of $C_{1}$ and $C_{2}$, respectively. The first of these is $G_{1}$ again. The second is $S\left(f p, \mathrm{Cl} D_{\alpha}^{*}\right)$, since none of the points in $C_{1}$ which are in the preimage of a point of $\mathrm{Cl}_{\alpha}^{*}$ can be permuted without moving points in the preimages of points outside $\mathrm{Cl} D_{\alpha}^{*}$.

The obvious homomorphism $H$ is suggested by the diagram

$$
G(p, M) \rightarrow\left[G(p, M) / G_{2} \otimes_{1} G_{1}\right] \rightarrow\left[G_{1} \approx G(f p, N) / S\left(f p, C_{1} D_{\alpha}^{*}\right)\right] .
$$

If $\operatorname{Cl} D_{\alpha}^{*}$ is nowhere dense in $M$, one can, for each $k$, let $N(k)=n(k)$, so that each of $G_{2}$ and $S\left(f p, C 1 D_{\alpha}^{*}\right)$ is the identity subgroup, and $H$ becomes an isomorphism. If $\mathrm{Cl}_{\alpha}^{*}$ is not nowhere dense, obvious examples show the above construction must fail to yield an isomorphism.

\section{References}

[1] R.D. Anderson, "The three conjugates theorem", (to appear). 
[2] Arnold R. Vobach, "On subgroups of the homeomorphism group of the Cantor set", Fund. Math. 60 (1967), 47-52.

[3] A.R. Vobach, "A theorem on homeomorphism groups and products of spaces", Bull. Austral. Math. Soc. 1 (1969), 137-141.

University of Houston,

Houston,

Texas, USA. 